\title{
Management of invasive aspergillosis in patients with COPD: Rational use of voriconazole
}

This article was published in the following Dove Press journal:

International Journal of COPD

23 July 2009

Number of times this article has been viewed

\section{Florence Ader' \\ Anne-Lise Bienvenu ${ }^{2}$ \\ Blandine Rammaert ${ }^{3}$ \\ Saad Nseir ${ }^{4}$}

'Service des Maladies Infectieuses et Tropicales; ${ }^{2}$ Service de Parasitologie et Mycologie Médicale, Université Claude Bernard Lyon I, Hospices Civils de Lyon, Hôpital de La CroixRousse, Lyon, France; ${ }^{3}$ Service des Maladies Infectieuses et Tropicales, Université Paris Descartes, Hôpital Necker Enfants Malades, Centre d'infectiologie Necker-Pasteur, Paris, France; ${ }^{4}$ Service de Réanimation Médicale, Hôpital A. Calmette, Centre Hospitalier Régional Universitaire, Lille, France
Correspondence: Florence Ader Service de Maladies Infectieuses et Tropicales - Hôpital de la Croix-Rousse, 104 Grande Rue de la Croix-Rousse, 69017 Lyon cedex 04, France

Tel +33472071963

Fax +33472 07I 750

Email florence.ader@univ-lyonl.fr

\begin{abstract}
Invasive pulmonary aspergillosis (IPA) is an important cause of mortality in patients with hematologic malignancies. The reported incidence of IPA in the context of chronic obstructive pulmonary disease (COPD) seems to increase. Approximately $1 \%-2 \%$ of overall fatal cases of IPA occur in COPD patients. The combination of factors such as lung immune imbalance, long-term corticosteroid use, increasing rate of bacterial exacerbations over time, and malnutrition are responsible for the emergence of IPA in these patients. The diagnosis of IPA is difficult to establish, which explains the delay in implementing accurate antifungal therapy and the high mortality rate. Persistent pneumonia nonresponsive to appropriate antibiotic treatment raises the concern of an invasive fungal infection. Definite diagnosis is obtained from tissue biopsy evidencing Aspergillus spp. on microscopic examination or in culture. Culture and microscopy of respiratory tract samples have a sensitivity and specificity of around $50 \%$. Other diagnostic tools can be useful in documenting IPA: computed tomography (CT) scan, nonculture-based tests in serum and/or in bronchoalveolar lavage such as antibody/antigen tests for Aspergillus spp. More recent tools such as polymerase chain reaction or [ $1 \rightarrow 3]-\beta-D$-glucan have predictive values that need to be further investigated in COPD patients. Antifungal monotherapy using azole voriconazole is recommended as a first-line treatment of IPA. This review assesses the use of voriconazole in COPD patients.
\end{abstract}

Keywords: chronic obstructive pulmonary disease, corticosteroid, Aspergillus, invasive pulmonary aspergillosis, voriconazole

\section{Introduction}

Invasive pulmonary aspergillosis (IPA) is a life-threatening pneumonia characterized by lung parenchyma invasion with vasculature erosion and necrosis that is caused by opportunistic fungi belonging to the species Aspergillus. Aspergillus fumigatus is the most common species recovered from cases of IPA. ${ }^{1}$ The major risk factor for IPA development is host immunosuppression, especially related to long-term systemic phagocytic depletion. Consequently, IPA has been strongly associated with hematological cancer patients (including recipients of hematopoietic stem cell transplants) despite efforts over the past two decades to reduce its incidence, and improve diagnosis and treatment methods. ${ }^{2}$ Other subsets of high-risk patients include solid-organ transplant recipients, patients with advanced HIV infection, inherited immunodeficiency, those receiving other immunosupresors such as monoclonal antibodies, and critically-ill patients. Of particular concern are patients who may not be obviously immunocompromised but combine lung immune imbalance with a systemic intrinsic and/or corticosteroid-induced immune dysfunction.

submit your manuscript | www.dovepress.con 
These patients do not strictly match with hematological cancer patient criteria regarding clinical presentation, radiological findings, and biological markers of infection. These include chronic obstructive pulmonary disease (COPD) patients that show the highest expansion in the number of reported cases over the past decade. ${ }^{3-8}$ COPD is a pulmonary disease characterized by airflow limitation that is not fully reversible. The airflow limitation is usually progressive and associated with an abnormal inflammatory response of the lung to noxious particles or gases. The chronic airflow limitation characteristic of COPD is caused by a mixture of small airway disease (obstructive bronchiolitis) and parenchymal destruction (emphysema), the relative contributions of which vary from person to person. Four stages of COPD are defined from mild, moderate, severe, to very severe (stages I to IV, respectively). ${ }^{9}$ Airway colonization by Aspergillus spp. is a common feature in chronic lung diseases. ${ }^{10}$ Although cumulative factors that trigger lung invasiveness are not completely understood, the leading concern remains when and how appropriate antifungal treatment should be initiated. This issue is critical considering the poor outcome unanimously reported in the literature of COPD patients suffering from IPA. ${ }^{5,6}$

Voriconazole is a synthetic antifungal agent belonging to the triazoles chemical family. Voriconazole has regulatory approval for primary treatment of IPA and has been recommended for use as the initial therapy for patients with this disease. ${ }^{2,11}$ This review provides an overview of the use of voriconazole and the particular aspects that have to be taken in consideration in the setting of COPD. This includes the need to refer to pathophysiological processes underlying IPA and to diagnostic issues that we will discuss as decisive supports for antifungal treatment initiation.

\section{Epidemiology}

An accurate estimation of IPA incidence in COPD patients is difficult, partly due to the lack of infection surveillance measures. In a study including 595 patients with IPA, 9\% suffered from nondetailed pulmonary diseases. ${ }^{12}$ In a review including 50 studies of aspergillosis case-fatality rate, COPD was the underlying condition in 26 out of 1941 patients $(1.3 \%)$ with aspergillosis. ${ }^{13}$ Several autopsy studies from intensive care unit (ICU) patients confirm that invasive fungal diseases (IFD) are among the most commonly missed diagnoses. ${ }^{14,15}$ Taken together, these studies suggest that IPA incidence amongst COPD patients may be underestimated. Higher risk of IPA occurrence may be correlated with advanced stages of COPD. As reported in a recent series of 57 probable IPA, 36 patients $(63.2 \%)$ were stage III and $21(33.8 \%)$ were stage IV. ${ }^{16}$ The global mortality rate of IPA in COPD patients reported in the literature is high, ranging from $72 \%$ to $95 \% .{ }^{5,6,16}$ This makes a sharp contrast with the latest outcome data from hematopoietic stem cell transplant recipients, where a clear trend toward a drop in attributable mortality has been observed in recent years (from $60 \%-70 \%$ to $40 \%$ ). ${ }^{17}$

\section{Pathophysiology}

Aspergillus spp. are airborne fungi that undergo a biphasicgrowth process. Spores or conidia, which represent the form of environmental persistence and dissemination, are inhaled during breathing and their small size $(2-3 \mu \mathrm{m})$ allows them to reach the lung alveoli. Conidia germinate to produce branching and septate filaments known as hyphae ( 2 to $10 \mu \mathrm{m}$ in diameter), which are the invasive form responsible for tissue damage. Upper respiratory tract clearance of inhaled conidia is facilitated by tracheobronchial ciliary activity. Conidia are ingested and destroyed by airway macrophages that prevent germination to hyphal form. Germinating spores and hyphae are attacked by polymorphonuclear neutrophils (PMNs) through the release of oxidants and degranulation. Pulmonary dendritic cells ingest both conidia and hyphae, migrate to draining lymph nodes and instruct the T helper-cell response. ${ }^{18}$

The development of IPA in COPD patients is due to combined factors. First, nutritional depletion is prevalent in advanced COPD and clinical research has provided evidence that undernutrition in advanced COPD is associated with an increased mortality, notably related to lung infections. ${ }^{19}$ A second factor is the impairment of ciliary function by chronic tobacco smoke and multiple episodes of infection reduce microbial pathogens clearance from lung airways. ${ }^{9}$ Third, the use of broad-spectrum antibiotics in case of exacerbations affects the distribution of normal flora, which may lead to a shift toward development of fungal populations in airways. Furthermore, a recent study showed that $A$. fumigatus has the ability to grow as a biofilm in vivo which may enable chronic persistence within lungs and promote antimicrobial resistance. ${ }^{20}$ This is of significant concern for COPD patients in ICU with synthetic devices in lung airways (eg, endotracheal tube). Finally, corticosteroids have significant impact on the distribution and function of neutrophils, monocytes, and lymphocytes. ${ }^{21,22}$ They also directly stimulate the growth of $A$. fumigatus in vitro possibly via the sterol binding protein in the fungus. ${ }^{23}$ Thus, intravenous (IV) corticosteroids treatment in COPD patients appears to be associated with a 
rising incidence of IPA. Some reports also describe that high doses of inhaled corticosteroids may promote the occurrence of IPA. ${ }^{24-26}$

In summary, COPD is characterized by significant variability in local immune balance that impacts the patient ability to contain invasive fungal challenges over time. Taken together, the intrinsic progression of the disease characterized by an increasing rate of microbial colonization, the exacerbations of viral and bacterial origins, the repetitive use of antibiotics, the necessity of occasional admissions in ICU with the requirement for invasive procedures, the steroid-induced immunomodulation, and the sepsis-related cycling fluctuations of immune status from hyper- to anti-inflammatory phases (that can confine to immunoparalysis in extreme cases $)^{27}$ create favorable conditions for the development of opportunistic invasive mould infections such as IPA.

\section{Clinical features}

The diagnosis of IPA in nonneutropenic patients is difficult because signs and symptoms are nonspecific such as fever, increased respiratory secretions, dyspnea exacerbation and high oxygen requirements, persistent bronchospasm despite elevated doses of corticosteroids. The occurrence of hemoptysis is less reported in COPD patients than in hematological patients. In the context of evolving COPD, persistent rapiddeveloping infiltrative abnormalities on thoracic imaging and/or persistent pulmonary infection despite broad-spectrum antibiotics should trigger further investigations focused on fungal infection.

\section{Assessing the diagnosis of IPA in COPD patients \\ Baseline criteria}

The definitions have assigned three levels of probability for the diagnosis of IPA: proven, probable, and possible. Proven IPA can apply to any patient, regardless of whether the patient is immunocompromised, on the common basis that histopathological documentation of infection and/or a culture positive specimen from a normally sterile site is required. This usually involves use of invasive procedures to obtain tissue specimens which is rarely feasible, especially in COPD patients.

In 2007, an attempt to standardize definitions of IPA in COPD patients was proposed by Bulpa and colleagues that established a formal framework based on previously published information. ${ }^{5}$ The classification focused on advanced stages of COPD (stages III and IV). The collection of hyphae in a lung biopsy specimen remained the decisive argument for proven IPA. For probable and possible IPA, the recommendation was given to rely on a combination of criteria taking into account nonspecific changes on chest computed tomography (CT) imaging, positive cultures from lower respiratory tract and positive biological markers such as serum antibody and/or antigen tests.

Definitions of IFD in immunocompromised patients have been recently revised. ${ }^{28}$ Aside from proven IPA, the definition of probable and possible IPA in immunocompromised patients is based on a combination of less-specific criteria within three categories: host factors, clinical manifestations (symptoms, signs, and radiological features), and mycological evidence. Probable IFD requires the presence of a host factor, a clinical criterion, and a mycological criterion. Cases that meet the criteria for a host and a clinical criterion but for which mycological evidence lacks are considered possible IFD. In this revised definition of IFD it has to be taken into account that predisposing host factors were extended to receipt of immunosuppressive agents including prolonged use of corticosteroids, which is often the case in COPD patients. However, the revised definitions do not apply to critically ill patients in the ICU who, nonetheless, may include COPD patients that develop probable or possible IPA.

\section{Diagnostic procedures}

\section{Lung biopsy}

Establishing the presence of fungi in tissue gives the highest level of certainty in diagnosing IPA. Transthoracic percutaneous needle aspiration, video-assisted thoracoscopic biopsy, or transbronchial biopsies are standard procedures to obtain histopathological evidence of IPA that reveals characteristic angular dichotomously branching septate hyphae on direct microscopic examination and/or Aspergillus spp. on culture. A yield in the range of $50 \%$ has been reported for percutaneous biopsy needle, which indicates that a negative result should not rule out the diagnosis. ${ }^{29}$ However, the invasiveness of these procedures exposes COPD patients to severe respiratory and/or bleeding complications.

\section{Microbiological examination Sputum}

Direct examination of sputum can be rapidly performed and is reported to be positive for Aspergillus in half of all IPA cases. $^{30}$ The use of fluorescence techniques optimizes the yield of microscopic examination up to $80 \%-90 \%{ }^{31}$

Based on literature data, the significance of positive sputum cultures for Aspergillus in COPD patients developing 
pneumonia is not clearly established. From a practical point of view, the possibility of IPA has to be taken into consideration in each case of advanced steroid-dependant COPD in patients experiencing antibiotic-resistant pneumonia. The recovery of several positive culture samples in a row in the course of such an ongoing pneumonia is clearly evocative of the diagnosis. In that case, further investigations must be triggered if possible and concern about antifungal treatment must be raised.

\section{Fiberoptic flexible bronchoscopy}

Fiberoptic flexible bronchoscopy with sampling of deep airway secretions and bronchoalveolar lavage (BAL) allows samples to be collected that can be processed for microscopic examination, fungal culture, and antigen or molecular detections. It has to be taken into account that BAL might be difficult to obtain in patients with low respiratory status due to advanced COPD. The macroscopic visualization of ulcerative lesions and/or pseudomembranes is suggestive of Aspergillus-related infection. The yield of culture positive specimens has ranged from $46 \%$ to $77 \% .^{30,32-35}$ BAL is more often positive in cases of prolonged pneumonia or extensive pulmonary lesions.

In summary, the isolation of Aspergillus spp. from the respiratory tract of a COPD patient with pneumonia confronts to two possibilities: colonization or current invasive disease. $\mathrm{BAL}$ is the technique that may provide the wider range of information through direct visualization of bronchial tree, and collection of distal samples. But BAL is often difficult to perform. Therefore, the diagnosis is more likely to rely on positive culture of sputum and bronchoaspirates. The positive predictive value for IPA of repeatedly positive sputum or bronchoaspirate cultures for Aspergillus spp. in advanced COPD patients must not be underestimated in the context of antibiotic-resistant pneumonia.

\section{Biological markers Detection of antibodies}

A positive serum antibody test for $A$. fumigatus remains one of the biological criteria proposed for the diagnosis of IPA in COPD according to Bulpa and colleagues. ${ }^{5}$ However, an increase in antibodies directed toward Aspergillus spp. requires a functional immune status and a pattern of infection long enough to generate a significant antibody response. Long-term steroid-dependant COPD patients are likely to develop weaker antibody responses and brief courses of IPA often do not allow seroconversion and the follow-up of kinetic antibody responses.
Antibody detection is more likely used in diagnosis of chronic pulmonary aspergillosis for which it is reported that high titers of antibodies are more common in more seriously ill patients, and generally would increase during exacerbations. ${ }^{36}$ On this basis, the monitoring of titers to detect the passage of chronic semi-invasive disease to invasive disease might be useful.

\section{Serological techniques}

The development of nonculture-based diagnostics has focused on the detection of surrogate markers for Aspergillus spp., such as the galactomannan (GM) antigen, $[1 \rightarrow 3]$ - $\beta$-D-glucan and the detection of Aspergillus DNA by polymerase chain reaction (PCR).

GM is a major Aspergillus cell-wall component that is released during the hyphal growth phase. It is used as an exo-antigen for the purpose of Aspergillus detection. ${ }^{37}$ The GM serum assay has been extensively investigated in immunocompromised patients. A meta-analysis including 27 studies regarding the value of the GM serum assay for surveillance of IPA in hematological and solid-organ transplant recipient patients ultimately revealed a moderate accuracy for diagnosis of IPA with various median sensitivity and specificity: for proven cases alone, which were $71 \%$ and $89 \%$ respectively; combining proven and probable cases, which were $61 \%$ and $93 \%$, respectively. ${ }^{38}$ The test is more useful in patients who have hematological malignancy or who have undergone hematopoietic cell transplantation than in solid-organ transplant recipients. The use of GM assay for the diagnosis of IPA in patients with COPD might be very limited. A retrospective study which enrolled critically ill patients without malignancy, $27 \%$ of whom were COPD patients, is worth mentioning: ${ }^{39}$ the diagnostic value of the GM assay demonstrated a sensitivity of only $53 \%$ in patients with proven or probable IPA (cut-off value of $1 \mathrm{ng} / \mathrm{mL}$ ). This indicates clearly that data from patients with malignancies and after solid organ transplantation cannot be extrapolated to other critical conditions. In the largest series available of IPA in COPD patients, $44 \%$ of the 34 patients tested were positive (cut-off value of $0.5 \mathrm{ng} / \mathrm{mL}$ ). ${ }^{16}$ The low sensitivity of GM in serum of these patients with values in a range of $40 \%-55 \%$ is a major limitation.

Because GM is a water-soluble carbohydrate, it can also be detected in BAL fluid. In small clinical studies among patients with hematological malignancies and solid organ transplant recipients, the sensitivity of the GM assay applied to BAL fluids ranges from $85 \%$ to $100 \%$ (cut-off value of $1.5 \mathrm{ng} / \mathrm{mL}) .{ }^{40-43}$ A recent prospective study conducted in ICU 
patients, of whom $8 \%$ were COPD patients, the sensitivity and specificity of GM detection in BAL fluid was $88 \%$ and $87 \%$, respectively (cut-off value of $0.5 \mathrm{ng} / \mathrm{mL}$ ). ${ }^{44}$ Consequently, there is an increasing tendency to use these samples for diagnosis of IPA despite the fact that the assay is not specifically validated for detection of GM in this fluid.

Several circumstances can be source of false-positives for the GM antigen test in either serum or BAL fluid. First, the false-positive reactivity can be caused by gastro-intestinal translocation of fungal GM from contaminated food or drink, ${ }^{45}$ or by the use of the intravenous antibiotics piperacillin-tazobactam and amoxicillin-clavulanic acid. ${ }^{46,47}$ Second, an important factor that affects the release of GM antigens is antifungal drug therapy. Different animal and human studies have shown decreased sensitivity of the GM assay when using prophylactic antifungal drugs. ${ }^{48,49}$

The $[1 \rightarrow 3]-\beta-D$-glucan is a cell wall component of many filamentous fungi and yeasts, including Aspergillus and Candida species. Reproducible assay results, with high specificity and a high positive predictive value, demonstrated that use of an assay to detect serum $[1 \rightarrow 3]-\beta$-D-glucan derived from fungal cell walls might be a useful diagnostic adjunct for invasive fungal infection. ${ }^{50} \mathrm{~A}$ single case of positive $[1 \rightarrow 3]-\beta$-D-glucan values during fatal IPA in a COPD patient has been reported which indicated a value increase at day 15 over the course of a 21 days-illness. ${ }^{51}$ In addition, falsepositive tests have been found in patients after hemodialysis, cardiopulmonary bypass surgery, high-dose immunoglobulin treatment, after exposure to glucan-containing gauze, and in cases of bacterial infections among ICU patients. ${ }^{52,53}$ Hence, the usefulness of $[1 \rightarrow 3]-\beta-D$-glucan in the diagnosis of IPA has clearly to be further evaluated.

Amplification of nucleic acid by PCR technology for the diagnosis of IPA is being increasingly studied. Yet, it is not included in the definitions of IPA due to the lack of standardization. It can be applied to serum and BAL specimens. ${ }^{54-59}$ Experience is still limited and a recent meta-analysis yielded a sensitivity and specificity for two consecutive positive samples of $75 \%$ and $85 \%$, respectively. ${ }^{60}$ However, most of studies were done in the hematological setting. Therefore, the role of PCR in patients with COPD is mostly unknown. As for the GM assay, there are factors that potentially have an impact upon the clinical sensitivity of PCR: the magnitude of the quantitative PCR signal decreases with antifungal therapy, potentially causing false-negative PCR results. ${ }^{61}$ The colonization of the respiratory tract by Aspergillus spp. may suggest a low positive predictive value. ${ }^{56}$ Finally, patients at risk for IPA are often prescribed a multitude of drugs and fluids, all of which may act as nonspecific inhibitors of the PCR. For example, anticoagulants inhibit PCR, thereby limiting its sensitivity. ${ }^{62}$

In summary, the use of GM, $[1 \rightarrow 3]-\beta$-D-glucan tests, and Aspergillus PCR as serological and molecular markers cannot be wisely advocated for routine use in COPD patients, and caution is warranted in the interpretation of positive test results in patients without a clinical suspicion of pulmonary infection and negative test results in patients with persisting pneumonia. The finding of sequentially positive GM tests in serum or BAL fluid together with a positive Aspergillus $\mathrm{PCR}$, in a patient with persisting pulmonary infection who carries one or more risk factors, is highly indicative for IPA and would justify considering antifungal therapy.

\section{Radiology}

Chest CT is an important tool for the diagnosis of IPA in neutropenic patients. CT has been proven effective in the absence of evident lesions on a conventional chest X-ray. Radiological findings might include nodules with rapid growth and/or cavitations. A "halo sign" (corresponding to a pulmonary mass surrounded by a zone of lower attenuation with ground-glass opacification produced by adjacent hemorrhage) and/or the "air crescent sign" (corresponding to crescentic radiolucencies around a nodular area of consolidation) may be present. ${ }^{63-71}$

However, thoracic imaging in mechanically ventilated ICU patients is less helpful due to many confounding factors such as atelectasis and, sometimes major, pleural effusions. A lower sensitivity (from $5 \%$ to $24 \%$ ) of the halo sign and air crescent sign in non-neutropenic patients has been reported in the literature. ${ }^{39,72,73}$ In the study by Guinea and colleagues, the worsening of imaging data is one of the five independent variables suggestive of IPA in patients with COPD and clinical isolation of Aspergillus spp. from lower respiratory samples. ${ }^{16}$

\section{Antifungal therapy}

In 2002, a large randomized trial reported an improved response and survival rate for patients with IPA who received therapy with voriconazole compared with patients who received standard deoxycholate amphotericin B therapy (so far, liposomal formulations of amphotericin B and voriconazole have not been compared in clinical trials for the treatment of IPA). This led to the recommendation to use voriconazole as the primary treatment of IPA. ${ }^{74}$ However, this study included 277 patients but only 16 were receiving corticosteroids as predisposing conditions; the majority of 
the remaining patients had hematological malignancies. Therefore, the evidence of the superiority of voriconazole over amphotericin B for the treatment of IPA in patients with COPD cannot be inferred from this study. Furthermore, rather conflicting data are available in experimental models. In a model of lethal infection in guinea pigs, two studies concluded that voriconazole therapy at $10 \mathrm{mg} / \mathrm{kg} /$ day prolonged survival compared to that of guinea pigs treated with conventional amphotericin B at 1 and $1.25 \mathrm{mg} / \mathrm{kg} / \mathrm{day}$, respectively. ${ }^{75,76}$ Another recent study concluded that the efficacy of high dose liposomal amphotericin B (at 5 and $10 \mathrm{mg} / \mathrm{kg}$ ) was superior to that of voriconazole $(10 \mathrm{mg} / \mathrm{kg})$ in a mouse model of pulmonary aspergillosis. ${ }^{77}$ Obviously, there is an urgent need for adequately-designed prospective clinical trials comparing voriconazole to conventional and/or liposomal formulation of amphotericin B in nonimmunocompromised high-risk patients, COPD inclusively.

Voriconazole is a synthetic triazole compound that exerts an inhibitory effect on two kinds of Aspergillus cytochrome P450-dependant enzymes: the first enzyme targeted is lanosterol 14- $\alpha$-demethylase that participates in ergosterol biosynthesis. Inhibition of this enzyme results in altered cell membrane function. The second group of targeted enzymes belongs to the fungal respiration chain. Their inhibition limits cell growth and replication and ultimately leads to cell death. Triazoles are generally considered as fungistatic.

Voriconazole is formulated as tablets or as sulfobutyl-ether cyclodextrin (SEC) solution for IV administration. SEC and voriconazole dissociate in plasma. As SEC is renally cleared and consequences of its plasma accumulation are still uncertain, caution is advised when using the IV formulation in patients with renal impairment (creatinine clearance $<50 \mathrm{~mL} / \mathrm{min}$ ). ${ }^{2,78}$ This concern does not apply to orally administrated voriconazole.

The oral formulation has $90 \%$ to $96 \%$ bioavailability. The absorption is better when administered either one hour before or after a meal. No formal relationship has been established between body weight and kinetics justifying the fixed dose in adult patient $\geq 40 \mathrm{~kg}$. Voriconazole is mostly hepatically metabolized, with only $5 \%$ of the drug appearing unchanged in the urine. The elimination half-life of $\sim 6$ hours warrants twice-daily dosing. Voriconazole is biotransformed by cytochrome P450-dependant isoenzymes CYP2C19, CYP2C9, and CYP3A4 acting as both a substrate and an inhibitor of these isoenzymes. This extensive and saturable hepatic metabolism explains the nonlinear pharmacokinetic profile of voriconazole in adults, with maximum concentration in plasma and area under the curve increasing disproportionally with increasing dose. Once voriconazole concentrations start approaching or exceeding the saturation level of the enzyme, small increases in the voriconazole dose will result in much larger increases in the drug concentration. ${ }^{79,80}$ Thus, intra- and interpatient voriconazole concentrations in serum may vary considerably depending on age, drug dose, concurrent illness, underlying liver function, drug-to-drug interactions, or genetic polymorphisms affecting cytochrome CYP2C19-mediated metabolism. ${ }^{78,81}$ Singlenucleotide polymorphisms contributing to slow metabolism are represented in $\sim 3 \%$ of white Europeans and in higher frequencies, from $15 \%$ to $20 \%$, among non-Indian Asian populations. ${ }^{82}$ An analysis that attempted to determine the relationship between voriconazole plasma concentrations and abnormal liver function test values, indicated that the risk of developing elevated liver function test values increased by $7 \%$ to $17 \%$ for every $1 \mu \mathrm{g} / \mathrm{mL}$ increase in the random voriconazole concentration. ${ }^{83}$ As no threshold was found, the investigators argued that therapeutic drug monitoring of voriconazole concentrations is not more helpful in predicting abnormal liver function test values than measuring liver function directly. Another recent study emphasizes that therapeutic drug monitoring of voriconazole improves the efficacy and safety of therapy in severely ill patients with invasive mycoses. Among the 52 enrolled patients, a large variability in voriconazole trough blood levels was observed, ranging from $<1 \mathrm{mg} / \mathrm{L}$ (the minimum inhibitory concentration at which, for most fungal pathogens, $90 \%$ of isolates are susceptible) to $>5.5 \mathrm{mg} / \mathrm{L}$ (the potentially toxic threshold).$^{84}$ Lack of response to therapy was more frequent in patients with low voriconazole levels $<1 \mathrm{mg} / \mathrm{L}$ (six patients, five of whom had IPA) than in those with voriconazole levels above that limit. In these patients, blood levels $>1 \mathrm{mg} / \mathrm{L}$ were reached after increasing the voriconazole dosage, with resolution of infection meaning that patients subsequently responded to therapy after their voriconazole daily dose was increased without the addition of a second antifungal to the treatment regimen. This result suggests that, although pharmacokinetic variability is a factor possibly contributing to the poor clinical outcome, dosage of voriconazole allows the correction of suboptimal serum levels. Among 16 patients with voriconazole trough blood levels $>5.5 \mathrm{mg} / \mathrm{L}$, five patients presented with neurological toxicity. In all cases, discontinuation of therapy resulted in prompt and complete neurological recovery. Thus, detection of voriconazole trough levels outside the therapeutic interval of $1-5.5 \mathrm{mg} / \mathrm{L}$ during the first week of therapy may help prevent treatment failures and anticipate the occurrence of drug-related toxicity.

Voriconazole diffuses widely in tissues with an emphasis on CSF levels which can reach up to $50 \%$ of plasma levels. Pharmacokinetics of voriconazole has been studied in 
conditions that might be relevant with COPD status: ${ }^{85}$ delivery of voriconazole by a jejunostomy twice daily appears feasible whether tablets are crushed or not. Consistently, nasogastric administration through nasogastric tube can be an alternative option to IV injection for mechanically ventilated patients although cautious has to be raised on intact intestinal absorption in this category of patients. Consequently, patient medications should be reviewed for drug interactions. Briefly, the main drugs that potentially increase voriconazole concentrations are erythromycin, indinavir, ranitidine, cimetidine, and omeprazole. Drugs that potentially decrease voriconazole concentrations are principally rifampicin (probably rifabutin as well) and phenytoin. Kinetics of other co-administrated drugs such as cyclosporine, digoxin, and phenytoin may be notably altered by voriconazole which implies adapting the dosages of some of these drugs based on the determination of their blood concentrations. ${ }^{85}$

Voriconazole's profile of adverse reactions include transient visual disturbances (principally photopsia), hepatotoxicity (elevated serum bilirubin, alkalin phosphatase, and hepatic aminotransferase enzyme levels) which may be dose limiting, and skin rash usually in sunlight-exposed areas. Visual and auditory hallucinations have been reported, mainly occurring upon receipt of initial loading doses ${ }^{86}$ Other side effects have been reported such as confusion, and pneumonitis. ${ }^{81,87}$

Treatment of IPA with voriconazole in adults is initiated with a loading dose of $6 \mathrm{mg} / \mathrm{kg}$ IV every 12 hours for two doses on the first day, followed by $4 \mathrm{mg} / \mathrm{kg}$ every 12 hours; ultimate oral dosage is $200 \mathrm{mg}$ every 12 hours. Primary combination therapy is not routinely recommended based on the lack of clinical data. Addition of another agent or switch to another drug class for salvage therapy may be considered in individual patient cases. Duration of therapy for most conditions associated with IPA has not been optimally defined. Duration should be from admission to treat until resolution or stabilization of all clinical and radiologic manifestations. Reversal of immunosuppression, if feasible, is important for a favorable outcome. The question whether long-term steroid treatment should be maintained, decreased, or interrupted should be addressed.

\section{Conclusion}

There are still many questions that need to be addressed and understood in the diagnosis and treatment of IPA in COPD patients. The high mortality rate is partly related to the delays in diagnosis and treatment because of non-specific symptoms and difficulties to meet gold standard criteria. A persistent pulmonary infection despite broad-spectrum antibiotics associated with abnormal CT thoracic imaging should trigger further investigations focused on moulds. Tissue biopsy evidencing Aspergillus spp. on microscopic examination or in culture is the gold standard but is difficult to obtain. Culture and microscopy of respiratory tract samples obtained by fiberoptic flexible bronchoscopy with sampling of deep airway secretions and BAL have an overall sensitivity and specificity of around 50\%. Combining noncultured-based diagnostic tools in serum and/or BAL (eg, PCR and GM or GM and [ $1 \rightarrow 3]-\beta$-D-glucan) is an important research direction that may improve the overall predictive value of these systems in patients. Antifungal therapy may be considered in COPD patients with rapidly progressive antibiotic-resistant pneumonia and should be started in that case when cultures for Aspergillus spp. or sequentially positive GM (in serum or BAL) are positive. The antifungal triazole voriconazole is recommended to be the first line treatment of IPA despite the lack of specific data in COPD patients. Based on the nonlinear metabolic elimination of this drug, a follow-up of voriconazole blood levels may be useful during the first week of therapy.

\section{Acknowledgments}

The authors gratefully acknowledge Dr. Andrée Hubber for providing useful comments.

\section{Disclosure}

The authors declare no conflicts of interest in this work.

\section{References}

1. Perfect JR, Cox GM, Lee JY, et al. The impact of culture isolation of Aspergillus species: A hospital-based survey of aspergillosis. Clin Infect Dis. 2001;33:1824-1833.

2. Walsh TJ, Anaissie EJ, Denning DW, et al. Treatment of aspergillosis: clinical practice guidelines of the Infectious Diseases Society of America. Clin Infect Dis. 2008;46:327-360.

3. Rello J, Esandi ME, Mariscal D, Gallego M, Domingo C, Valles J. Invasive pulmonary aspergillosis in patients with chronic obstructive pulmonary disease: report of eight cases and review. Clin Infect Dis. 1998;26:1473-1475.

4. Ader F, Nseir S, Le Berre R, et al. Invasive pulmonary aspergillosis in chronic obstructive pulmonary disease: an emerging fungal pathogen. Clin Microbiol Infect. 2005;11:427-429.

5. Bulpa P, Dive A, Sibille Y. Invasive pulmonary aspergillosis in patients with chronic obstructive pulmonary disease. Eur Respir J. 2007;30:782-800

6. Samarakoon P, Soubani AO. Invasive pulmonary aspergillosis in patients with COPD: a report of five cases and systematic review of the literature. Chron Respir Dis. 2008;5:19-27.

7. Meersseman W, Lagrou K, Maertens J, Van Wijngaerden E. Invasive aspergillosis in the intensive care unit. Clin Infect Dis. 2007;45:205-216.

8. Bouza E, Guinea J, Peláez T, Pérez-Molina J, Alcalá L, Muñoz P. Workload due to Aspergillus fumigatus and significance of the organism in the microbiology laboratory of a general hospital. J Clin Microbiol. 2005;43:2075-2079.

9. Rabe KF, Hurd S, Anzueto A, et al; Global Initiative for Chronic Obstructive Lung Disease. Global strategy for the diagnosis, management, and prevention of chronic obstructive pulmonary disease: GOLD executive summary. Am J Crit Care Respir Med. 2007;176:532-555. 
10. Yu VL, Muder RR, Poorsattar A. Significance of isolation of Aspergillus from the respiratory tract in diagnosis of invasive pulmonary aspergillosis. Results from a three-year prospective study. Am J Med. 1986;81:249-254.

11. Steinbach WJ, Stevens DA. Review of newer antifungal and immunomodulatory strategies for invasive aspergillosis. Clin Infect Dis. 2003; 37(Suppl 3):S157-S187.

12. Patterson TF, Kirkpatrick WR, White M, et al. Invasive aspergillosis. Disease spectrum, treatment practices, and outcomes. I3 Aspergillus Study Group. Medicine (Baltimore). 2000;79:250-260.

13. Lin SJ, Schranz J, Teutsch SM. Aspergillosis case-fatality rate: systematic review of the literature. Clin Infect Dis. 2001;32:358-366.

14. Roosen J, Frans E, Wilmer A, Knockaert DC, Bobbaers H. Comparison of premortem clinical diagnoses in critically iII patients and subsequent autopsy findings. Mayo Clin Proc. 2000;75:562-567.

15. Dimopoulos G, Piagnerelli M, Berre J, Eddafali B, Salmon I, Vincent JL. Disseminated aspergillosis in intensive care unit patients: an autopsy study. J Chemother. 2003;15:71-75.

16. Guinea M, Torres-Narbona P. Gijon T. Pelaez P. Munoz J. De Miguel E, Bouza E. Invasive pulmonary aspergillosis in patients with COPD: A description of 57 cases collected in a single tertiary hospital (1999-2008). Abstract M-2161. Washington, D.C: 48th ICAAC/IDSA 46th Annual Meeting; October 25-28, 2008.

17. Upton A, Kirby KA, Carpenter P, Boeckh M, Marr KA. Invasive aspergillosis following hematopoietic cell transplantation: outcomes and prognostic factors associated with mortality. Clin Infect Dis. 2007;44:531-540.

18. Romani L. Immunity to fungal infections. Nat Immunol. 2004;4:1-13.

19. Landbo C, Prescott E, Lange P, Vestbo J, Almdal TP. Prognostic value of nutritional status in chronic obstructive pulmonary disease. Am J Respir Crit Care Med. 1999;160:1856-1861.

20. Seidler MJ, Salvenmoser S, Müller FMC. Aspergillus fumigatus forms biofilms with reduced antifungal drug susceptibility on bronchial epithelial cells. Antimicrob Ag Chemoter. 2008;52:4130-4136.

21. Schaffner A. Therapeutic concentrations of glucocorticoids suppress the antimicrobial activity of human macrophages without impairing their responsiveness to gamma interferon. J Clin Invest. 1985;76:1755-1764.

22. Diamond RD. Inhibition of monocyte-mediated damage to fungal hyphae by steroid hormones. J Infect Dis. 1983;147:160.

23. Ng TTC, Robson GD, Denning DW. Hydrocortisone enhanced growth of Aspergillus spp: implications for pathogenesis. Microbiology. 1994;140:2475-2479.

24. Peter E, Bakri F, Ball DM, Cheney RT, Segal BH. Invasive pulmonary filamentous fungal infection in a patient receiving inhaled corticosteroid therapy. Clin Infect Dis. 2002;36:54-56.

25. Barouky R, Badet M, Saint Denis M, Soubirou JL, Philit F, Guerin C. Inhaled corticosteroids in COPD and disseminated aspergillosis. Eur J Int Med. 2003;14:380-382.

26. Cornet M, Hallat H, Somme D, et al. Fulminant invasive pulmonary aspergillosis in immunocompetent patients. A two-case report. Clin Microbiol Infect. 2003;9:1224-1227.

27. Hartemink KJ, Paul MA, Spijkstra JJ, Girbes ARJ, Polderman KH. Immunoparalysis as a cause of invasive aspergillosis? Int Care Med. 2003;29:2068-2071.

28. De Pauw B, Walsh TJ, Donnelly JP, et al. Revised definitions of invasive fungal disease from the European Organization for Research and Treatment of Cancer/Invasive Fungal Infections Cooperative Group and the National Institute of Allergy and Infectious Diseases Mycoses Study Group (EORTC/MSG) Consensus Group. Clin Infect Dis. 2008; 46:1813-1821.

29. Jantunen E, Piilonen A, Volin L, et al. Diagnostic aspects of invasive Aspergillus infections in allogeneic BMT recipients. Bone Marrow Transplant. 2000;25:867-871.

30. Kahn FW, Jones JM, England DM. The role of bronchoalveolar lavage in the diagnosis of invasive pulmonary aspergillosis. Am J Clin Pathol. 1986;86:518-523.

31. Andreas S, Heindl S, Wattky C, Möller K, Rüchel R. Diagnosis of pulmonary aspergillosis using optical brighteners. Eur Respir J. 2000;15:407-411.
32. Caillot D, Mannone L, Cuisenier B, Couaillier JF. Role of early diagnosis and aggressive surgery in the management of invasive pulmonary aspergillosis in neutropenic patients. Clin Microbiol Infect. 2001;7:54-61.

33. Horvath JA, Dummer S. The use of respiratory-tract cultures in the diagnosis of invasive pulmonary aspergillosis. Am J Med. 1996;100:171-178.

34. Kaiser L, Huguenin T, Lew PD, Chapuis B, Pittet D. Invasive aspergillosis. Clinical features of 35 proven cases at a single institution. Medicine (Baltimore). 1998;77:188-194.

35. Levy H, Horak DA, Tegtmeier BR, Yokota SB, Forman SJ. The value of bronchoalveolar lavage and bronchial washings in the diagnosis of invasive pulmonary aspergillosis. Respir Med. 1992;86:243-248.

36. Denning DW, Riniotis K, Dobrashian R, Sambatakou H. Chronic cavitary and fibrosing pulmonary and pleural aspergillosis: case series, proposed nomenclature change, and revew. Clin Infect Dis. 2003;37(S3): S265-S280.

37. Maertens J, Verhaegen J, Lagrou K, Van Eldere J, Boogaerts M. Screening for circulating galactomannan as a noninvasive diagnostic tool for invasive aspergillosis in prolonged neutropenic patients and stem cell transplantation recipients: a prospective validation. Blood. 2001;97:1604-1610.

38. Pfeiffer CD, Fine JP, Safdar N. Diagnosis of invasive aspergillosis using a galactomannan assay: a meta-analysis. Clin Infect Dis. 2006;42: 1417-1427.

39. Meersseman W, Vandecasteele SJ, Wilmer A, Verbeken E, Peetermans WE, Van Wijngaerden E. Invasive aspergillosis in critically ill patients without malignancy. Am J Respir Crit Care Med. 2004;170:621-625.

40. Sanguinetti M, Posteraro B, Pagano L, et al. Comparison of real-time PCR, conventional PCR, and galactomannan antigen detection by enzyme-linked immunosorbent assay using bronchoalveolar lavage fluid samples from hematology patients for diagnosis of invasive pulmonary aspergillosis. J Clin Microbiol. 2003;41:3922-3925.

41. Musher B, Fredricks D, Leisenring W, Balajee SA, Smith C, Marr KA. Aspergillus galactomannan enzymenimmunoassay and quantitative PCR for diagnosis of invasive aspergillosis with bronchoalveolar lavage fluid. J Clin Microbiol. 2004;42:5517-5522.

42. Seyfarth HJ, Nenoff P, Winkler J, Krahl R, Haustein UF, Schauer J. Aspergillus detection in bronchoscopically acquired material. Significance and interpretation. Mycoses. 2001;44:356-360.

43. Clancy CJ, Jaber RA, Leather HL, et al. Bronchoalveolar lavage galactomannan in diagnosis of invasive pulmonary aspergillosis among solid-organ transplant recipients. J Clin Microbiol. 2007;45:1759-1765.

44. Meersseman W, Lagrou K, Maertens J, et al. Galactomannan in bronchoalveolar lavage fluid: a tool for diagnosing aspergillosis in intensive care unit patients. Am J Respir Crit Care Med. 2008;177:27-34.

45. Ansorg R, Van den Boom R, Rath PM. Detection of Aspergillus galactomannan antigen in foods and antibiotics. Mycoses. 1997;40:353-357.

46. Sulahian A, Touratier S, Ribaud P. False positive test for Aspergillus antigenemia related to concomitant administration of piperacillin and tazobactam. N Engl J Med. 2003;349:2366-2367.

47. Viscoli C, Machetti M, Cappellano P, et al. False-positive galactomannan platelia Aspergillus test results for patients receiving piperacillin-tazobactam. Clin Infect Dis. 2004;38:913-916.

48. Becker MJ, De MS, Fens MH, Verbrugh HA, Bakker-Woudenberg IA. Effect of amphotericin B treatment on kinetics of cytokines and parameters of fungal load in neutropenic rats with invasive pulmonary aspergillosis. J Antimicrob Chemother. 2003;52:428-434.

49. Marr KA, Laverdiere M, Gugel A, Leisenring W. Antifungal therapy decreases sensitivity of the Aspergillus galactomannan enzyme immunoassay. Clin Infect Dis. 2005;40:1762-1769.

50. Ostrosky-Zeichner L, Alexander BD, Kett DH, et al. Multicenter clinical evaluation of the [1->3]- $\beta$-D-glucan assay as an aid to diagnosis of fungal infections in humans. Clin Infect Dis. 2005;41:654-659.

51. Okada S, Teramoto S, Takizawa H, Ouchi Y, Matsuoka R. Clinical insignificance of $1->3$ ]- $\beta$-D-glucan in early diagnosis of invasive pulmonary aspergillosis in a patient with chronic obstructive pulmonary disease. J Med Microb. 2003;52:1031-1032.

52. Vandewoude KH, Vogelaers D, Blot SI. Aspergillosis in the ICU-the new 21st century problem? Med Mycol. 2006;44:S71-S76. 
53. Digby J, Kalbfleisch J, Glenn A, Larsen A, Browder W, Williams D. Serum glucan levels are not specific for presence of fungal infections in intensive care unit patients. Clin Diagn Lab Immunol. 2003;10:882-885.

54. Skladny H, Buchheidt D, Baust C, et al. Specific detection of Aspergillus species in blood and bronchoalveolar lavage samples of immunocompromised patients by two-step PCR. J Clin Microbiol. 1999;37:3865-3871.

55. Bretagne S, Costa JM, Marmorat-Khuong A, et al. Detection of Aspergillus species DNA in bronchoalveolar lavage samples by competitive PCR. J Clin Microbiol. 1995;33:1164-1168.

56. Raad I, Hanna H, Sumoza D, Albitar M. Polymerase chain reaction on blood for the diagnosis of invasive pulmonary aspergillosis in cancer patients. Cancer. 2002;94:1032-1036.

57. Raad I, Hanna H, Huaringa A, Sumoza D, Hachem R, Albitar M. Diagnosis of invasive pulmonary aspergillosis using polymerase chain reaction-based detection of Aspergillus in BAL. Chest. 2002;121:1171-1176.

58. Buchheidt D, Baust C, Skladny H, Baldus M, Brauninger S, Hehlmann R. Clinical evaluation of a polymerase chain reaction assay to detect Aspergillus species in bronchoalveolar lavage samples of neutropenic patients. Br J Haematol. 2002;116:803-811.

59. White PL, Linton CJ, Perry MD, Johnson EM, Barnes RA. The evolution and evaluation of a whole blood polymerase chain reaction assay for the detection of invasive aspergillosis in hematology patients in a routine clinical setting. Clin Infect Dis. 2006;42:479-486.

60. Mengoli C, Cruciani M, Barnes RA, Loeffler J, Donnelly P. Use of PCR for diagnosis of invasive aspergillosis: systematic review and meta-analysis. Lancet Infect Dis. 2009;9:89-96.

61. Hope WW, Walsh TJ, Denning DW. Laboratory diagnosis of invasive aspergillosis. Lancet Infect Dis. 2005;5:609-622.

62. Garcia ME, Blanco JL, Caballero J, Gargallo-Viola D. Anticoagulants interfere with PCR used to diagnose invasive aspergillosis. J Clin Microbiol. 2002;40:1567-1568.

63. Collins J. CT signs and patterns of lung disease. Radiol Clin North Am. 2001;39:1115-1135.

64. Caillot D, Couaillier JF, Bernard A, et al. Increasing volume and changing characteristics of invasive pulmonary aspergillosis on sequential thoracic computed tomography scans in patients with neutropenia. J Clin Oncol. 2001;19:253-259.

65. Greene R. The radiological spectrum of pulmonary aspergillosis. Med Mycol. 2005;43:S147-S154.

66. Horger M, Einsele H, Schumacher U, et al. Invasive pulmonary aspergillosis: frequency and meaning of the "hypodense sign" on unenhanced CT. Br J Radiol. 2005;78:697-703.

67. Kami M, Kishi Y, Hamaki T, et al. The value of the chest computed tomography halo sign in the diagnosis of invasive pulmonary aspergillosis. An autopsy-based retrospective study of 48 patients. Mycoses. 2002;45:287-294.

68. Lee YR, Choi YW, Lee KJ, Jeon SC, Park CK, Heo JN. CT halo sign: the spectrum of pulmonary diseases. Br J Radiol. 2005;78:862-865.

69. Pasmans HL, Loosveld OJ, Schouten HC, Thunnissen F, van Engelshoven JM. Invasive aspergillosis in immunocompromised patients: findings on plain film and (HR) CT. Eur J Radiol. 1992;14:37-40.
70. Won HJ, Lee KS, Cheon JE, et al. Invasive pulmonary aspergillosis: prediction at thin-section CT in patients with neutropenia - a prospective study. Radiology. 1998;208:777-782.

71. Pinto PS. The CT halo sign. Radiology. 2004;230:37-40.

72. Vandewoude KH, Blot SI, Benoit D, Colardyn F, Vogelaers D. Invasive aspergillosis in critically ill patients: attributable mortality and excesses in length of ICU stay and ventilator dependence. J Hosp Infect. 2004;56:269-276.

73. Greene RE, Schlamm HT, Stark P, et al. Radiological findings in acute invasive pulmonary aspergillosis: utility and reliability of halo sign and air crescent sign for diagnosis and treatment of invasive pulmonary aspergillosis in high-risk patients [abstract]. European Congress of Microbiology and Infectious Diseases, 13th annual meeting; 2003.

74. Herbrecht R, Denning DW, Patterson TF, et al. Voriconazole versus amphotericin B for primary therapy of invasive aspergillosis. $N \mathrm{Engl}$ J Med. 2002;347:408-415.

75. Chandrasekar PH, Curtright J, Manavathu E. Efficacy of voriconazole against invasive pulmonary aspergillosis in a guinea-pig model. $J$ Antimicrob Chemother. 2000;45:673-676.

76. Kirkpatrick WR, McAtee RK, Fothergill AW, Rinaldi MG, Patterson TF. Efficacy of voriconazole in a guinea pig model of disseminated invasive aspergillosis. Antimicrob Agents Chemother. 2000; 50:2865-2868.

77. Takemoto K, Yamamoto Y, Ueda Y, Kanazawa K, Yoshida K, Niki Y. Comparative study on the sfficacy of liposomal amphotericin B and voriconazole in a murine pulmonary aspergillosis model. Chemotherapy. 2009;55:105-113.

78. Zonios DI, Bennett JE. Update on azole antifungals. Semin Respir Crit Care Med. 2008;29:198-210.

79. Drusano GL. How does a patient maximally benefit from anti-infective chemotherapy? Clin Infect Dis. 2004;39:1245-1246.

80. Lewis RE. What is the "therapeutic range" for voriconazole. Clin Infect Dis. 2008;46:212-214.

81. Boyd AE, Modi S, Howard SJ, Moore CB, Keevil BG, Denning DW. Adverse reactions to voriconazole. Clin Infect Dis. 2004;39:1241-1244.

82. Balian JD, Sukhova N, Harris JW, et al. The hydroxylation of omeprazole correlates with S-mephenytoin metabolism: a population study. Clin Pharmacol Ther. 1995;57:662-669.

83. Lutsar I, Hodges MR, Tomaszewski K, Troke PF, Wood ND. Safety of voriconazole and dose individualization. Clin Infect Dis. 2003;36: 1087-1088.

84. Pascual A, Calandra T, Bolay S, Buclin T, Bille J, Marchetti O. Voriconazole therapeutic drug monitoring in patients with invasive mycoses improves efficacy and safety outcomes. Clin Infect Dis. 2008;46:201-211.

85. Levêque D, Nivoix Y, Jehl F, Herbrecht R. Clinical pharmacokinetics of voriconazole. Int J Antimicrob Agents. 2006;27:274-284.

86. Zonios DI, Gea-Banacloche J, Childs R, Bennett JE. Hallucinations during voriconazole therapy. Clin Infect Dis. 2008;47:e7-e10.

87. Boucher HW, Groll AH, Chiou CC, Walsh TJ. Newer systemic antifungal agents: pharmacokinetics, safety and efficacy. Drugs. 2004;64:1997-2020.
International Journal of COPD

\section{Publish your work in this journal}

The International Journal of COPD is an international, peer-reviewed journal of therapeutics and pharmacology focusing on concise rapid reporting of clinical studies and reviews in COPD. Special focus is given to the pathophysiological processes underlying the disease, intervention programs, patient focused education, and self management protocols.

\section{Dovepress}

This journal is indexed on PubMed Central, MedLine and CAS. The manuscript management system is completely online and includes a very quick and fair peer-review system, which is all easy to use. Visit http://www.dovepress.com/testimonials.php to read real quotes from published authors. 